\title{
Optimization of Degree Regenced Segment Lines Detection Using Ant Colony Optimization Algorithm
}

\author{
Johnie Rogers Swanda Pasaribu ${ }^{1}$, Elviawaty M. Zamzami ${ }^{1}$, Pahala Sirait ${ }^{2}$, \\ A M H Pardede ${ }^{3}$ and M Heikal $^{4}$ \\ \{johniepasaribu@students.usu.ac.id\} \\ ${ }^{1}$ Department of Computer Science,Universitas Sumatera Utara, Medan, Indonesia \\ ${ }^{2}$ Department of Informatics, STMIK Mikroskil, Medan, Indonesia \\ ${ }^{3}$ STMIK Kaputama, Jl. Veteran No. 4A-9A, Binjai, Sumatera Utara, Indonesia \\ ${ }^{4}$ Department of Management, Universitas Malikussaleh, Aceh, Indonesia
}

\begin{abstract}
Edge detection is an important operation in digital image analysis to get a better image as needed. In the edge detection process to determine the location of the points that are the edge of the object. The purpose of edge detection is to detect borders that constrain two areas of image, object and background. In this paper will be done pre-image processing by eliminating noise using Geometric Mean Filter algorithm then will be done edge detection using compass operators and will be calculated MSE and PSNR, then as a comparison with line detection with Hough Transform algorithm and edge detection with Ant Colony algorithm. The results obtained are reduced error and increase Peak to Noise Ratio (PSNR).
\end{abstract}

Keywords: Edge detection, Ant Colony optimization, Compass operator

\section{Introduction}

To get the image information analyzed, an image edge detection process is required. Until now, it is known that image edge detection method is still less than optimal because it produces incomplete, unclear, or disconnected image edges. Therefore, an image edge detection method should be developed so that the information intensity is incomplete because the edges of the disconnected image can be reduced. Ant Colony Optimization (ACO) is an optimization algorithm based on ant colony behavior. ACO uses a probabilistic approach to the graph problem. By using $\mathrm{ACO}$, the problem of calculating process duration in determining the optimal path in terms of graph can be reduced. ACO is the optimal path search method on parallel search-based graphs and each agent has the ability to view and remember it.

\section{Methods}




\subsection{Edge Detection}

The edge of the image is a change in the intensity value of gray/fast (large) at close range. While the edge detection in the image is an automated process that can recognize the edges of the image object. Edge detection is an operation performed to detect edges that limit two different areas of homogeneous imagery [1]. Edge detection on images is a process that produces the edges of a drawing object, which aims to:

1. To mark the part that becomes the detail of the image. 2. Fixed the details of blurred images, which occurred due to errors or effects of the image acquisition process.

3. And change the 2D image into curve shape.

\subsection{Compass Operator}

The compass operator is a routine operator used in image processing problems. this operator performs gradient calculations on 8 wind direction by using convolution matrix S, N, SE, NW, W, E, SW and NE.

\subsection{Ant Colony Optimization (ACO) algorithm}

The working principle of ACO is that the ant always leaves a special chemical (pheromone) on the path through it during the trip [3]. The pheromone left by the ant at a point it passes will be used by the ant behind to guide it on the way. The more ants that pass through these pathways the more pheromones will be, so the possibility of other ants following the path will be greater. Furthermore, the pheromone left by the ants on the road will evaporate over time.

\subsection{Hough Transformation}

The Hough Transformation was first introduced by Paul Hough in 1962 to detect a straight line. Hough Transformation is a picture transformation technique that can be used to isolate or in other words acquire the image feature. Since the purpose of transformation is to get more specific features, Classical Hough Transform is the most common technique used to detect curved objects such as lines, circles, ellipses and parabolas. The main advantage of the Hough transform is that it can detect a margin with a gap at the feature boundary and is relatively unaffected by noise or noise. By using the parametric form fromHough transform and uses the poll to determine the exact parameter value. If in the picture there are several lines intersecting at one point, then when the point is transformed into the parameter space $\mathrm{m}-\mathrm{c}$ it will be found that the transformation on the parameter space $\mathrm{m}-\mathrm{c}$ is a straight line with the line.

\subsection{The Scheme of System}

To maximize the results of image detection, then performed advanced image processing of edge detection results with Ant Colony Optimization algorithm (ACO). Prior to edge detection, the input image is first processed to remove noise using the Geometric Mean Filter algorithm and add contrast with the Histogram Equalization algorithm. Then the image of the processing and the image of the edge detection results are modeled into an ant colony. Transforming the input image's result to the ant colony will be optimized with ACO. The transformed colony is then transformed back into an output image. The tested images are evaluated for performance using 
ground truth imagery.Compatible edge detection performance of Compass operator and line edge detection with Hough Transform and ACO algorithms are evaluated based on the results of image processing and ground truth imagery.

In this study the algorithm for image processing of edge detection results is Ant Colony Optimization (ACO) with the following stages:

a. Reading of image pixel values

Before the image processing done, firstly done pixel image value reading which likes true color 24 bit image. The reading serves to create the red, green and blue (RGB) color matrix images.

b. Preprocessing the image.

Prior to edge detection, the input image is first processed by increasing its contrast with the Histogram Equalization algorithm and eliminating noise using the Geometric Mean Filter algorithm.

c. Image edge detection with the Compass operator.

In the image prapengolahan done edge detection with the Kompas operator which aims to reinforce the edges of objects in the image. Edge detection operator This compass uses the eight-point wind kernel.

d. Detect lines and circles on the image with the Hough Transform algorithm

In the image edge detection results with the operator Compass done detection lines and circles to make improvements to the lines and circles of objects on the image is disconnected (degraded)

e. Image Edge Detection with ACO Algorithm

In the process of image edge detection with ACO algorithm, the input image needs to be transformed into a graph. ACO algorithm is only able to do optimization if the problem is graph. The two-dimensional image input image is converted to gray image. Then the pixel value is taken to be a graph node. The node represents the places that ants can visit that have the corresponding pixel information. The node is connected to neighboring pixels according to the original image. Of the overall pixels that have been converted to nodes, are evaluated to determine the endpoint. At each endpoint is placed one ant for the initial location of the graph search. Then created tabulist which is the visibility matrix of paths that exist between the nodes. The value of the tabulist determines which node each destination will move

f. In image edge detection result with ACO algorithm is calculated value of MSE and PSNR that is difference value of initial image before processed with image result of process.

\subsection{Mean Square Error (MSE) and Peak Signal to Noise Ratio (PSNR)}

The MSE in the statistics is the mean square of error which is the difference between the original image and the processing image. The smaller the MSE value the similarity level between the original image and the higher processing image the MSE is used to measure the average error derived from the amount to be estimated. The formula for calculating MSE can be seen as equation 2.9 [4].

$\mathrm{MSE}=\frac{1}{N} \sum_{i=1}^{n}\left(S-S_{e}\right)^{2}$ 


\subsection{Data Set}

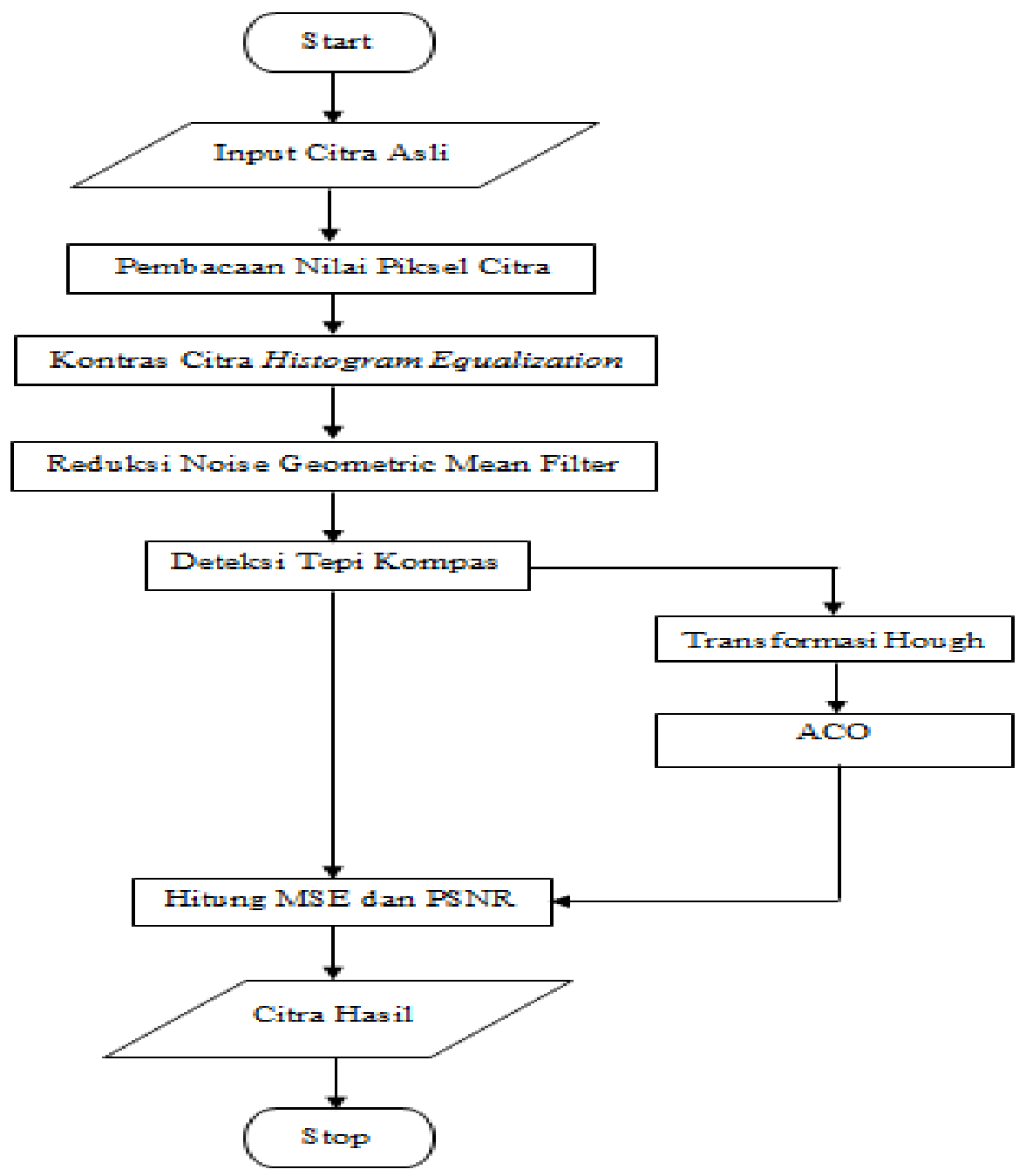

\section{Result}

Figure 1. Dataset 
The result of image segmentation test with the ant colony optimization algorithm is a test with $400 \times 300$ pixel image dimension which yields mse, psnr and segmented image.

The mse and pnsr values of the image segmentation using ant colony optimization can be seen in the following table:

Table 1. The threshold values of MSE and PSNR Compass Edge Detection Algorithm

\begin{tabular}{|c|c|c|c|c|}
\hline \multirow[t]{2}{*}{ No } & \multirow{2}{*}{$\begin{array}{l}\text { File } \\
\text { Cover }\end{array}$} & \multirow{2}{*}{$\begin{array}{c}\text { Size } \\
\text { (kbyte) }\end{array}$} & \multicolumn{2}{|c|}{ Parameter } \\
\hline & & & MSE & PSNR \\
\hline 1 & Image 1 & 30,95 & 14783,98 & 17,63 \\
\hline 2 & Image 2 & 55,39 & 5326,37 & 13,20 \\
\hline 3 & Image 3 & 25,54 & 10956,15 & 16,33 \\
\hline 4 & Image 4 & 147,71 & 11577,97 & 16,57 \\
\hline 5 & Image 5 & 9,97 & 6791,76 & 14,25 \\
\hline 6 & Image 6 & 48,67 & 14652,47 & 17,59 \\
\hline 7 & Image 7 & 19,93 & 6418,92 & 14,00 \\
\hline 8 & Image 8 & 38,71 & 19274,65 & 18,78 \\
\hline 9 & Image 9 & 22,85 & 4584,58 & 12,55 \\
\hline 10 & Image 10 & 55,29 & 5387,97 & 13,25 \\
\hline & Average & & 9975,48 & 15,41 \\
\hline
\end{tabular}

Table 2. The threshold values of MSE and PSNR The Result of Combination Algorithm

\begin{tabular}{|ccccc|} 
No & $\begin{array}{c}\text { File } \\
\text { Cover }\end{array}$ & $\begin{array}{c}\text { Size } \\
(\text { kbyte })\end{array}$ & MSE & \multicolumn{2}{c|}{ Parameter } \\
\hline $\mathbf{1}$ & Image 1 & 30,95 & 0,1183 & 33,3323 \\
\hline $\mathbf{2}$ & Image 2 & 55,39 & 0,1244 & 33,1161 \\
\hline $\mathbf{3}$ & Image 3 & 25,54 & 0,1094 & 33,6752 \\
\hline $\mathbf{4}$ & Image 4 & 147,71 & 0,1348 & 32,7680 \\
\hline $\mathbf{5}$ & Image 5 & 9,97 & 0,1150 & 33,4552 \\
\hline $\mathbf{6}$ & Image 6 & 48,67 & 0,1225 & 33,1812 \\
\hline $\mathbf{7}$ & Image 7 & 19,93 & 0,1169 & 33,3839 \\
\hline $\mathbf{8}$ & Image 8 & 38,71 & 0,1153 & 33,4458 \\
\hline $\mathbf{9}$ & Image 9 & 22,85 & 0,1411 & 32,5680 \\
\hline $\mathbf{1 0}$ & Image 10 & 55,29 & 0,1169 & 33,3867 \\
\hline & Average & 0,1214 & 33,2312 \\
\hline
\end{tabular}

\section{Conclusions}


From the results of the research, it can be seen that the results by using the compass algorithm only are very different from using a combination algorithm that is the compass algorithm, hough transformation and ant colony algorithm, thus from Mean Square Error (MSE) and Peak Signal to Noise Ratio (PSNR) the combination algorithm can be seen better.

\section{References}

[1] Čisar, P., Čisar, S.M \& Markoski, B. 2013. Kernel Sets in Compass Edge Detection. CINTI 2013 14th IEEE International Symposium on Computational Intelligence and Informatics • 19-21 November, 2013 - Budapest, Hungary. Academy of Criminalistic and Police Studies, Belgrade-Zemun, Serbia.

[2] Dhiman, D \& Hooda R. 2014. An Enhanced Ant Colony Optimization Based Image Edge Detection. Department of ECE Department of ECE. International Journal of Advanced Research in Computer Science and Software Engineering Research Paper Volume 4, Issue 6, June 2014 ISSN: 2277 128X IET, Bhaddal, India IET, Bhaddal, India.

[3] Dorigo, M, Birattari, M. \& Thomas. 2006. Ant Colony Optimization Artificial Ants as a Computational Intelligence Technique, IEEE Computational Intelligence Magazine Nov 2006.

[4] Gonzalez, R.C. \& Woods, R.E. Digital Image Processing. second edition, USA: Addison-Wesley Publishing Co, University of Tennessee.

[5] Gonzalez, R.C. \& Woods, R.E. Digital Image Processing. third edition, USA: Addison-Wesley Publishing Co, University of Tennessee.

[6] Gupta, C \& Gupta S. 2013. Edge Detection of an Image based on Ant Colony Optimization Technique. International Journal of Science and Research (IJSR), India Online ISSN. Manav Rachna International University. India.

[7] Moparthi, R. 2015. Speed Optimized Implementation Of Ant Colony Optimization Algorithm For Image Edge Detection. A Paper Submitted to the Graduate Faculty of the North Dakota State University of Agriculture and Applied Science.

[8] Panetta, K., Fellow, Gao, C., Agaian, S. \& Nercessian, S. 2015. A New Reference-Based Edge Map Quality Measure. International Journal. IEEE Transactions On Systems, Man, And Cybernetics: Systems.

[9] Ping, G, Chunbo, X, Cheng Yi \& Jing. L. 2014. Adaptive Ant Colony Optimization Algorithm. 2014 International Conference on Mechatronics and Control (ICMC) July 3 - 5, 2014, Jinzhou, China. IEEE.

[10] Swiatnicki, Z. 2015. Application of Ant Colony Optimization Algorithms for Transportation Problems Using the Example of the Travelling Salesman Problem. 4th IEEE International Conference on Advanced logistics and Transport (ICAl T). Logistics Department Faculty of National Security and Logistics Polish Air Force Academy Dyblin, Poland.

[11] Tu, L \& Dong, C. 2013. Histogram Equalization and Image Feature Matching. 2013 6th International Congress on Image and Signal Processing (CISP 2013). School of Science, University of Science and Technology Liaoning province, China.

[12] Vallepalli, S. S \& Rajendran, M. M. 2012. Image De-noising using Mean Pixel Algorithms Corrupted with Photocopier Noise. International Journal. University of Michigan - (Ann Arbor; Dearborn) Signal and Image Processing laboratory - UM-Ann Arbor Digital Forensics laboratory - UM-Dearborn. IWSSIP 2012, 11-13 April 2012, Vienna, Austria.

[13] Venmathi, A.R., Ganesh E.N \& Kumaratharan, N. 2016. Kirsch Compass Kernel Edge Detection Algorithm for Micro Calcification Clusters in Mammograms. Middle-East Journal of Scientific Research 
[14] Yelmanova, E \& Romanyshyn, Y. 2017. Histogram-based Method for Image Contrast Enhancement. Department of ECT, Lviv Polytechnic National University, UKRAINE, University of Warmia and Mazury in Olsztyn, Poland.

[15] Zhu, Y \& Huang, C. 2012. An Adaptive Histogram Equalization Algorithm on the Image Gray Level Mapping. 2012 International Conference on Solid State Devices and Materials Science. College of Electronic Information Engineering Jiangsu Teachers University of Technology Changzhou, China. 